\title{
Energy Level Modulation of HOMO, LUMO, and Band- Gap in Conjugated Polymers for Organic Photovoltaic Applications
}

\author{
Bong-Gi Kim, Xiao Ma, Chelsea Chen, Yutaka le, Elizabeth W. Coir, Hossein Hashemi, \\ Yoshio Aso, Peter F. Green, John Kieffer, and Jinsang Kim*
}

To devise a reliable strategy for achieving specific HOMO and LUMO energy level modulation via alternating donor-acceptor monomer units, we investigate a series of conjugated polymers (CPs) in which the electron withdrawing power of the acceptor group is varied, while maintaining the same donor group and the same conjugated chain conformation. Through experiment and DFT calculations, good correlation is identified between the withdrawing strength of the acceptor group, the HOMO and LUMO levels, and the degree of orbital localization, which allows reliable design principles for CPs. Increasing the acceptor strength results in an enhanced charge transfer upon combination with a donor monomer and a more pronounced decrease of the LUMO level. Moreover, while HOMO states remain delocalized along the polymer chain, LUMO states are strongly localized at specific bonds within the acceptor group. The degree of LUMO localization increases with increasing polymer length, which results in a further drop of the LUMO level and converges to its final value when the number of repeat units reaches the characteristic conjugation length. Based on these insights we designed PBT8PT, which exhibits $6.78 \%$ power conversion efficiency after device optimization via the additive assisted annealing, demonstrating the effectiveness of our predictive design approach.
Dr. B.-G. Kim, Prof. P. F. Green, Prof. J. Kim Macromolecular Science and Engineering University of Michigan

Ann Arbor, Michigan 48109, USA

E-mail: jinsang@umich.edu

X. Ma, Dr. C. Chen, E. W. Coir, H. Hashemi,

Prof. P. F. Green, Prof. J. Kieffer, Prof. J. Kim

Department of Materials Science and Engineering

University of Michigan

Ann Arbor, Michigan 48109, USA

Prof. Y. le, Prof. Y. Aso

The Institute of Scientific and Industrial Research

Osaka University

8-1 Mihogaoka, Ibaraki, Osaka 567-0047, Japan

Prof. Y. le

JST-PREST, Kitakyushu Science and Research Park

2-5 Hibikino, Wakamatsu-ku, Kitakyushu city 808-0135, Japan

Prof. P. F. Green, Prof. J. Kim

Department of Chemical Engineering

University of Michigan

Ann Arbor, Michigan 48109, USA

DOI: 10.1002/adfm.201201385

\section{Introduction}

Organic photovoltaic cells incorporating conjugated polymers (CPs) have drawn great attention as a cost-effective alternative to silicon-based solar cells. The power conversion efficiency (PCE) of these devices has enhanced rapidly during the last couple of years and currently exceeds $6 \% .{ }^{[1-6]}$ This dramatic improvement of OPV performance could be ascribed to the development of new materials, mostly $\mathrm{CPs}$, and to the growing understanding of OPV device operation..$^{[1-11]}$ One of the fundamental requirements for photovoltaic energy conversion is the collection of as much sunlight as possible. In this regard, CP's absorption range should match the high photon flux region of the solar spectrum, thus the ability to tailor the optical band-gap of CPs for OPV devices is crucially important for efficient solar energy harvesting. Nevertheless, effective device operation requires the CP's energy levels to move in opposition to a reduced bandgap: CPs should have a low highest occupied molecular orbital (HOMO) to help increase the open circuit voltage $\left(V_{o c}\right)^{[12]}$ and a high lowest unoccupied molecular orbital (LUMO), compared to the fullerene's LUMO, for efficient charge dissociation in the $\mathrm{CP} / \mathrm{PCBM}$ bulk heterojunction device.

The most promising strategy for tailoring the $\mathrm{CP}$ band-gap involves introducing donor (D) and acceptor (A) monomers together in the polymer framework. ${ }^{[13-14]}$ The underlying concept is that the interaction between alternating electron-rich donors and electron-deficient acceptors results in a compressed band-gap via molecular orbital hybridization and via intramolecular charge transfer. ${ }^{[15]} \mathrm{A}$ drawback of this method, however, is that too strong an acceptor in the CP's framework could deteriorate its hole transporting property ${ }^{[16]}$ or could generate an extremely low LUMO in the $\mathrm{CP}^{[17]}$ resulting in poor charge dissociation. It is therefore clear that achieving specific HOMO and LUMO energy levels with precision, as well as controlling the band-gap, is essential for effective material development.

The hybridization of molecular orbitals upon combining various monomeric groups results in energy levels that are 
not easily predictable. This is because the HOMO/LUMO energy levels strongly depend on the degree of electron delocalization within the CP's effective conjugation length (ECL), and the latter is resolutely affected by the backbone's conformation. ${ }^{[18-20]}$ Hence, the magnitude of the energy gap in D-A type CPs cannot be predicted simply based on the HOMO and LUMO levels of the isolated monomers to be combined in the $\mathrm{CP}$, but the monomers' intrinsic attributes such as shape, side chains, and functional groups affecting the backbone conformation must be taken into account. In this study, four different CPs were designed and synthesized in order to identify a way to modulate and precisely predict the HOMO and LUMO of CPs. The band-gap narrowing was systematically explored by monitoring the energy level trends of applied monomers and resulted CPs. A detailed interpretation of the observed trends was accomplished through complementary first-principles calculations. In addition, optical and electrical properties of obtained CPs were directly correlated with OPV performance, and an example CP (designated PBT8PT) obtained from the energy level modulation was suggested and investigated under different device fabrication conditions to provide a way to optimize the OPV device.

\section{Results and Discussion}

A comparison of the HOMO/LUMO levels of our series of CPS with those of their constituent monomers is only meaningful if all CPs have a similar chain conformation. As shown in Scheme 1, we designed and synthesized analogous D-A structured CPs which all have the same donor, 4,8-bis(2-ethylhexyloxy)benzo[1,2-b;3,4-b]dithiophene. The CPs' main frames were constructed solely via thiophene derivatives to ensure a rigidplanar conformation. ${ }^{[21,22]}$ Quantum mechanical calculations (Figure 1) revealed that all of our polymers have exactly the same zig-zag shaped back-bone geometry. Although PBTCT and PBTCTO exhibit twist angles between the D-A repeat units of $7.5^{\circ}$ and $7.9^{\circ}$, respectively, which are slightly larger than for the other polymers $\left(\approx 1^{\circ}-3^{\circ}\right)$, they are further planarized in the film state (PBTCT showed a $40 \mathrm{~nm}$ red-shift in its absorption $\lambda_{\max }$

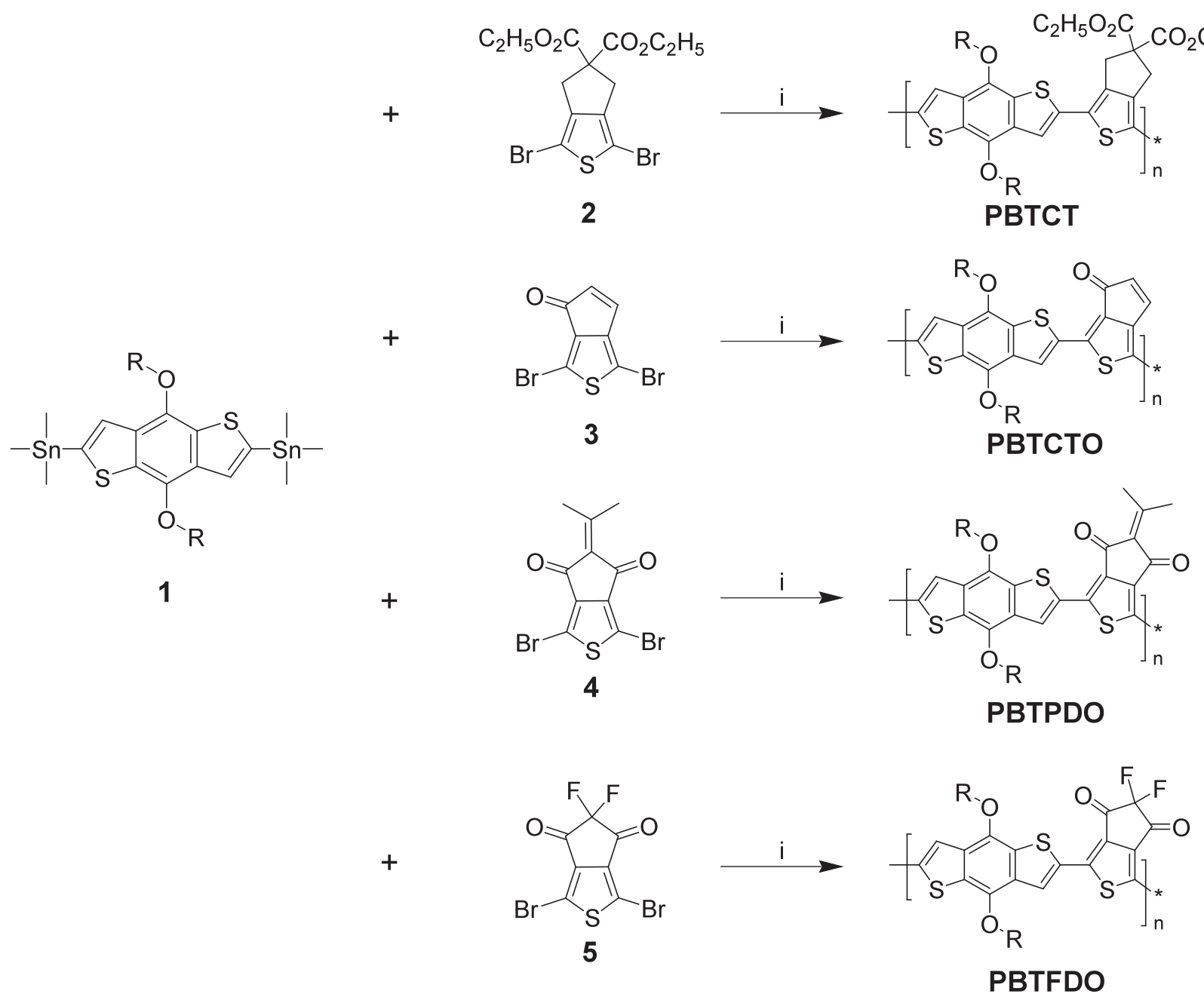

Scheme 1. Detailed synthetic scheme of designed CPs. R indicates 2-ethylhexyl group and i) $\mathrm{Pd}_{2}(\mathrm{dba})_{3}, \mathrm{P}(\mathrm{o} \text {-tolyl })_{3}$, toluene, $110^{\circ} \mathrm{C}, 48 \mathrm{~h}$. 


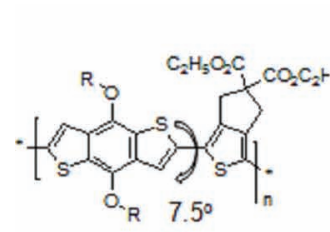

PBTCT

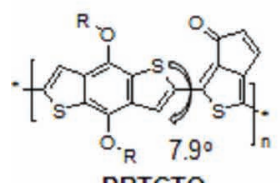

РBTCTO

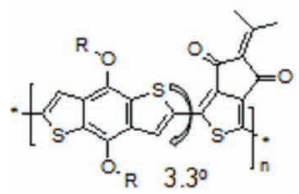

PBTPDO

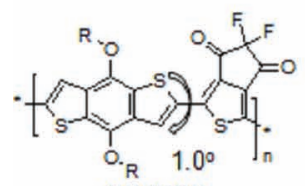

PBTFDO
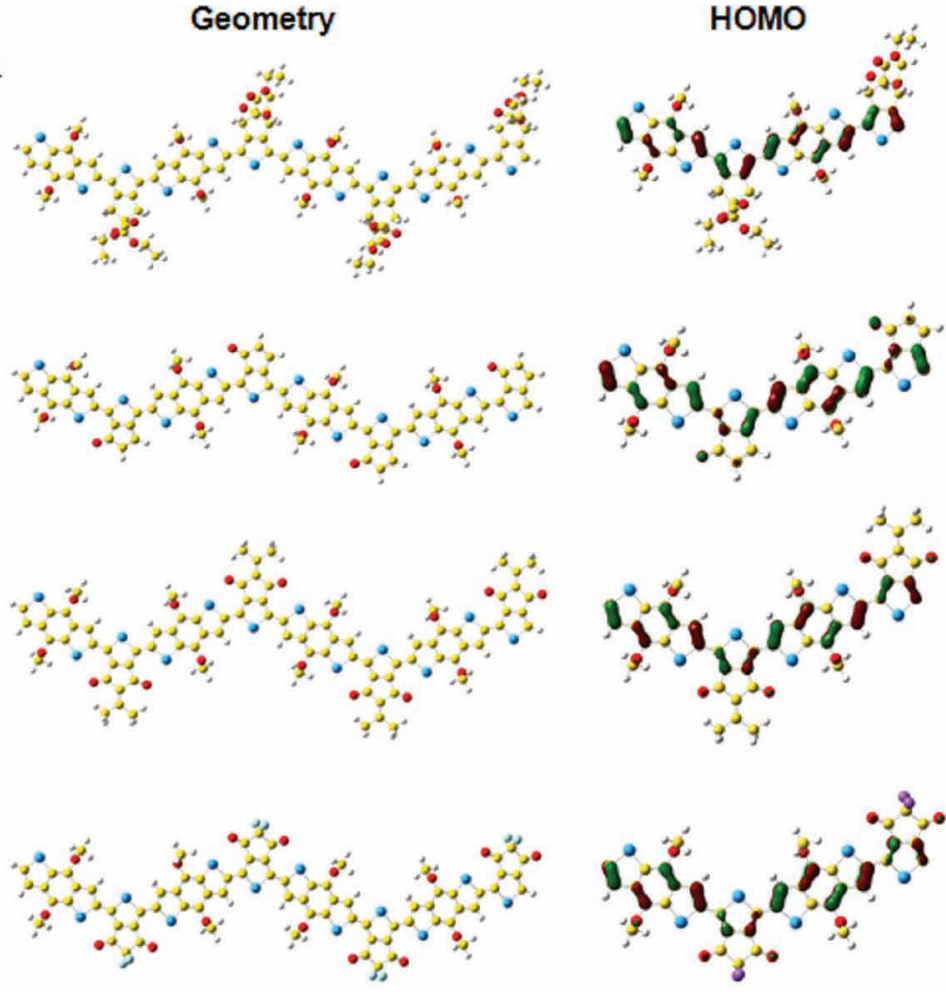
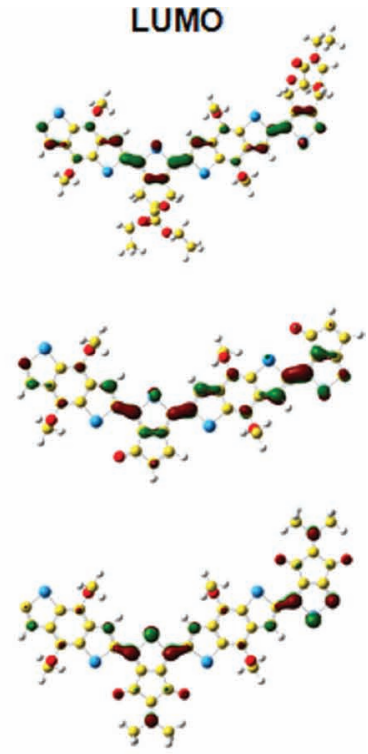

\begin{tabular}{l} 
E \\
\hline \\
\hline \\
\hline
\end{tabular}

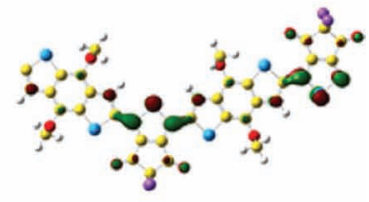

Figure 1. Chain conformations of designed CPs were expected from their tetramer's conformation calculation under minimized energy state, and their dimer's electron distributions both in HOMO/LUMO state are illustrated. R is 2-ethylhexyl group and it was abbreviated as methyl group for the calculation work.

in solid state compared to its corresponding solution). Thus, all CPs in this study have similar rigid-planar chain conformations in solid state and can be expected to have similar ECLs.

The influence of the electron accepting group on the CPs' absorption characteristics was then studied with UV-vis spectroscopy. As depicted in Figure 2a, the absorption of the CPs red-shifts as stronger acceptor monomers are combined with the same donor monomer. The absorption edge shifts from $600 \mathrm{~nm}$ in PBTCT to $825 \mathrm{~nm}$ in PBTFDO, reflecting the narrowing of the optical band-gap in this sequence. As shown in Figure $2 \mathrm{~b}$, the color of the CPs changes from orange (PBTCT) to purple (PBTCTO), to blue (PBTPDO) and to greenish blue (PBTFDO), due to the absorption edge shift. To understand the underlying principles that govern this development, we begin by comparing the energy levels of the CPs we synthesized, with those of their constituent D and A groups. In this study, the CPs' energy levels were directly measured by means of cyclic voltammetry (CV) as shown in Figure S1 (Supporting Information). Furthermore, we used DFT calculations to determine the HOMO and LUMO levels of the isolated D and A monomers and of CPs containing 2, 3, 4, and an infinite number of repeat units, the latter being achieved by constructing a simulation cell that is periodic in the direction of the polymer backbone (Figure S2a, Supporting Information). DFT calculations reveal that upon combining a donor and an acceptor group into a single D-A repeat unit, the resulting HOMO level approximately corresponds to that of the donor group and the LUMO level to that of the acceptor group. Upon combining several of these (a)

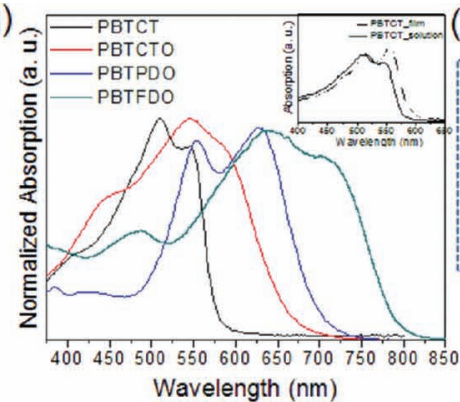

(b)

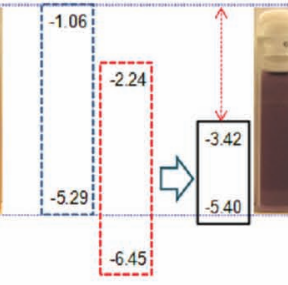

РвтСто

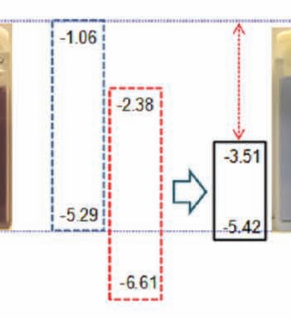

PBTPDO

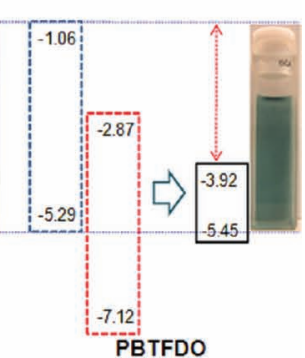

PBTFDO

Figure 2. a) Absorption spectra of obtained CPs in chloroform solution, and b) energy level diagrams of applied monomers and obtained CPs. All drawn energy levels are proportional to the scale of the absolute energy level (dashed blue boxes-donor monomers, dashed red boxes- acceptor monomers and solid black boxes-polymers). 
(a)
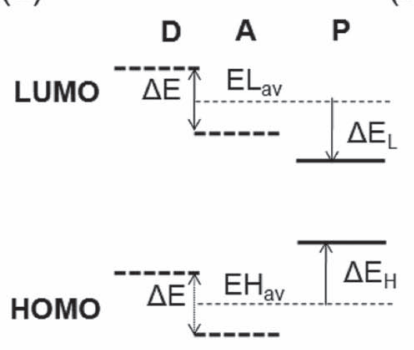

$\mathrm{HOMO}(\mathrm{P})=\mathrm{EH}_{\mathrm{av}}-\Delta \mathrm{E}_{\mathrm{H}}$ LUMO $(P)=E_{a v}-\Delta E_{L}$

b)

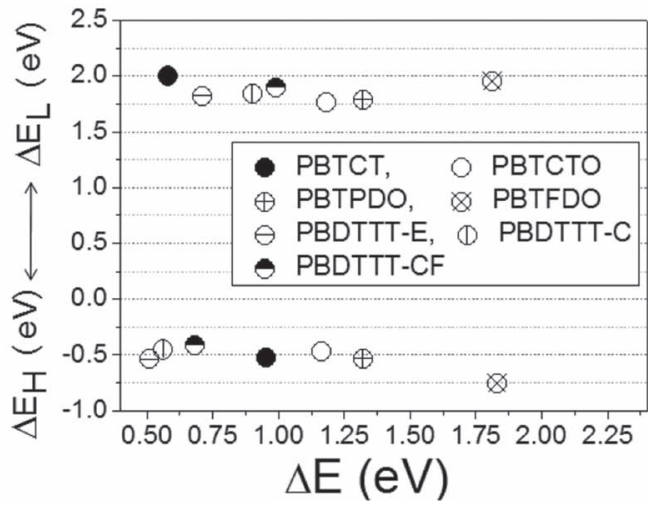

Figure 3. a) Energy level diagram and correlation equation. The dashed line indicates the average energy level of donor (D) monomer and acceptor (A) monomer, and $\mathrm{P}$ indicates the resulting polymer. b) Analyzed energy level correlation both from our series of CPs and three reported CPs in the literature. ${ }^{[1]}$

repeat units the HOMO levels decrease only very little, for all CPs investigated, while the LUMO levels decrease more prominently. Conversely, the more electronegative the constituents of the acceptor group, the more pronounced the decrease in the LUMO level, ultimately leading to a narrowing of the energy band gap in the sequence from PBTCT to PBTFDO, as shown in Figure $2 \mathrm{~b}$. This trend is echoed by the localization behavior of electronic orbitals. While for all polymers the HOMO remains delocalized along the polymer backbone, the LUMO become increasingly localized with increasing chain length and the withdrawing strength of the acceptor. For example, in PBTCT, significant LUMO density can be found within the thiophene units of donor and acceptor monomers, while the LUMO is strongly concentrated within the connecting bonds between donor and acceptor and the sulfur atom of the acceptor in PBTFDO. The electron localization induces intramolecular charge separation, which likely endows an elevated electron affinity to CPs via the electron deprived moieties, comparable to the trend of CP's LUMO level decrease when a stronger electron withdrawing monomer was incorporated. A similar trend has been observed for a D-A type copolymers: as the degree of polymerization increases, the LUMO level decreases more rapidly than the HOMO level due to molecular orbital hybridization of each monomer, finally producing a narrowed band gap. ${ }^{[23]}$ Based on first-principles calculations, it was shown that there is a strong effect of the degree of LUMO localization on the energy gap. ${ }^{[23]}$ For our system, the fact that the HOMO levels of the CPs are essentially controlled by that of the constituent donor group, ${ }^{[24]}$ provides a reliable strategy for designing CPs that exhibit a higher $V_{\mathrm{oc}}$

Further analysis of the relation between the energy levels of our CPs and those of their monomers reveals an interesting feature. To this effect we define $\Delta E_{\mathrm{H}}$ as the change in energy from the midpoint between the HOMO levels of the isolated donor and acceptor monomers to the HOMO level of the resulting D-A polymer, and $\Delta E_{\mathrm{L}}$ as the corresponding construct for the LUMO levels. As shown in Figure 3, $\Delta E_{\mathrm{H}}$ and $\Delta E_{\mathrm{L}}$, each are essentially constant for all CPs we synthesized, and amount to $-0.5 \mathrm{eV}$ and $1.9 \mathrm{eV}$, respectively. To confirm the ubiquity of this observation, we analyzed several CPs (PBTTTT-E, PBTTTT$\mathrm{C}$ and PBTTTT-CF) that likely have a chain conformation ${ }^{[1]}$ similar to those of the CPs in this study in the same way. These materials exhibit the same trends, with $\Delta E_{\mathrm{H}}$ and $\Delta E_{\mathrm{L}}$ values differing by $\pm 0.1 \mathrm{eV}$ from the aforementioned ones. Since molecular orbital hybridization between the monomers during the polymerization occurs only within the CP's ECL, CPs with analogous chain conformation should have a similar ECL and are expected to retain a comparable energy level mutation from those of their own monomeric repeating units. In order to investigate the effect of the CP's conformation on $\Delta E_{\mathrm{H}}$ or $\Delta E_{\mathrm{L}}$, another series of $\mathrm{CPS}^{[25-28]}$ that shares chemical structure similarities but whose conformations differ from those of the CPs studied above were analyzed, as shown in Figure S3 (Supporting Information). The applied energy levels of each monomer unit are summarized in Figure $\mathrm{S} 2 \mathrm{~b}$ (Supporting Information). These CPs exhibit different values of $\Delta E_{\mathrm{H}}$ and $\Delta E_{\mathrm{L}}$ compared to the series of CPs described in this contribution, but still show the same constancy in HOMO and LUMO level shifts, i.e., $\Delta E_{\mathrm{H}}=-0.9 \mathrm{eV}$ and $\Delta E_{\mathrm{L}}=1.5 \mathrm{eV}$. Although the same values of $\Delta E_{\mathrm{H}}$ and $\Delta E_{\mathrm{L}}$ cannot be universally applicable to all D-A type CPs, within a given family of compounds this design concept can be adopted to predict $\mathrm{CP}$ energy levels from their constituent monomers with reasonable accuracy, as long as they maintain an analogous chain conformation. To compare OPV performance depending on the modulated energies of our CPs, we fabricated OPV devices. The results are summarized in Table 1. All devices were made after blending the polymer solution (10 mg into $2 \mathrm{~mL}$ of o-dichlorobenzene) with PCBM[70] (10 mg) because the CPs had poor solubility (likely due to their rigidplanar conformation in spite of their low molecular weights $\left(\mathrm{M}_{\mathrm{n}}\right.$; 10 500-13 $300 \mathrm{~g} / \mathrm{mol})$ ). All devices made with our CPs worked

Table 1. Summary of the properties of the OPV devices obtained with the synthesized CPs using different process conditions.

\begin{tabular}{|c|c|c|c|c|c|c|c|c|}
\hline Sample & РВТСТ & РВТСТО & PBTPDO & PBTFDO & & & & \\
\hline Applied method & additive & additive & additive & additive & heat & none & additive & additive heat \\
\hline Layer thickness & $80 \mathrm{~nm}$ & $80 \mathrm{~nm}$ & $80 \mathrm{~nm}$ & $80 \mathrm{~nm}$ & $120 \mathrm{~nm}$ & $120 \mathrm{~nm}$ & $120 \mathrm{~nm}$ & $120 \mathrm{~nm}$ \\
\hline$J_{\mathrm{sc}}\left(\mathrm{mA} / \mathrm{cm}^{2}\right)$ & -3.39 & -8.76 & -10.68 & -1.20 & -10.03 & -10.27 & -12.37 & \\
\hline$V_{o c}(V)$ & 0.62 & 0.72 & 0.73 & 0.79 & 0.85 & 0.91 & 0.88 & \\
\hline Fill Factor & 0.53 & 0.62 & 0.62 & 0.45 & 0.49 & 0.48 & 0.62 & $\mathrm{~N} / \mathrm{A}$ \\
\hline PCE (\%) & 1.13 & 3.90 & 4.84 & 0.27 & 4.22 & 4.48 & 6.78 & \\
\hline
\end{tabular}


(a)

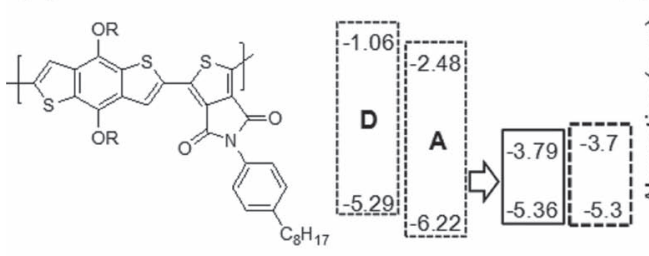

(c)

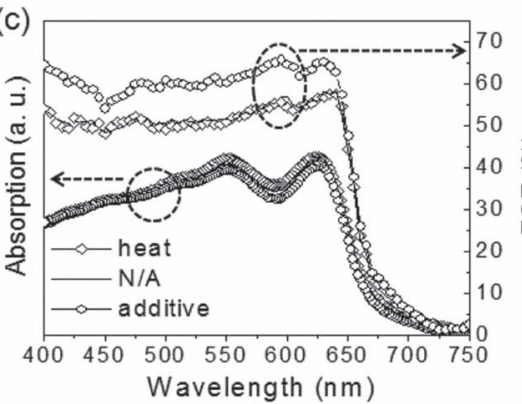

(b)

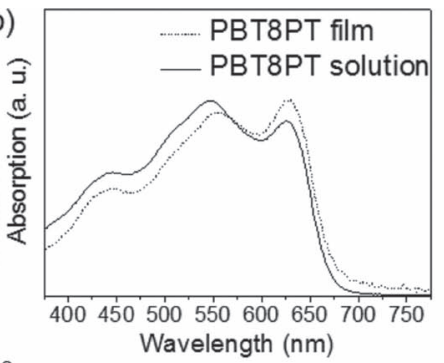

(d)

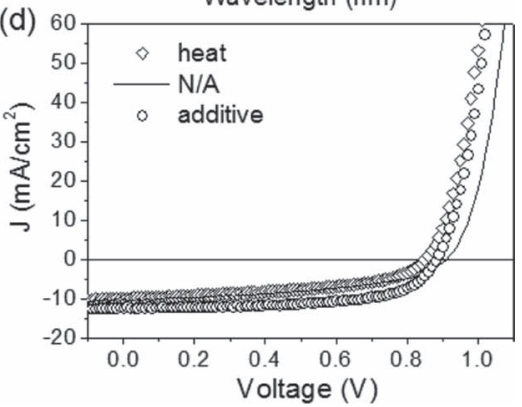

Figure 4. a) Chemical structure of PBT8PT (R; 2-ethylhexyl) and energy diagram (dashed blue box: donor monomer; dashed red box: acceptor monomer; solid black box: polymer from CV; and dashed black box: polymer from calculation). b) Absorption spectra in solution $\left(\mathrm{CHCl}_{3}\right)$ and dried film. c) Absorption spectra of obtained devices and $\mathrm{EQE}$, and d) OPV device performance.

successfully, except for PBTFDO because its LUMO level $(3.9 \mathrm{eV})$ is too low for PCBM[70] to dissociate excitons. This emphasizes how important selecting a proper acceptor monomer is when designing D-A type CPs. As the CP's absorption shifts to a longer wavelength region from PBTCT to PBTPDO, photocurrent generation improves due to its absorption adjustment in the high photo-flux region $(600-800 \mathrm{~nm})$. The $V_{\text {oc }}$ increases as the CP's HOMO level decreases, as explained by the modified Shockley equation. ${ }^{[12]}$ Among the CPs tested in this study, PBTPDO exhibits the best OPV performance with about 5\% PCE, because it has a good absorption region to harvest solar radiation and because its lower HOMO level results in a higher $V_{\mathrm{oc}}$. Although their energy levels (including the optical band-gap) were systematically controlled, the OPV performance from our series of CPs still lags behind reported CPs having similar absorption region. ${ }^{[1,4]}$ We believed that this is due to a low molecular weight which is not helpful for long range percolation pathways for charge carrier transfer ${ }^{[29,30]}$ and due to a poor solubility which limits an active layer thickness $(80 \mathrm{~nm})$ for better light harvesting.

To demonstrate the reliability of the established energy level prediction and to further improve OPV device performance, a new CP (PBT8PT, Figure 4a), which is expected to have a rigidplanar conformation similar to that of the four CPs studied in this contribution, was designed. An extra octyl side chain was introduced to increase the molecular weight through improved solubility. As shown in Figure 4b, PBT8PT exhibits nearly the same absorption properties both in solution $\left(\mathrm{CHCl}_{3}\right)$ and in the dried film, indicating a rigid-planar polymer conformation. We predicted PBT8PT's energy levels assuming $-0.5 \mathrm{eV}$ for $\Delta E_{\mathrm{H}}$ and $1.9 \mathrm{eV}$ for $\Delta E_{\mathrm{L}}$. As shown in Figure 4a, these predicted energy levels (based on the monomers' energy levels confirmed with $\mathrm{CV}$ ) agree well with the measured values using CV. Although the LUMO level showed slightly larger deviation $(0.09 \mathrm{eV})$ than

the HOMO level $(0.04 \mathrm{eV})$, our results demonstrate that $\Delta E_{\mathrm{H}}$ and $\Delta E_{\mathrm{L}}$ still converge to the same values if the $\mathrm{CP}$ conformation is similar. When OPV devices were fabricated with newly devised PBT8PT under various process conditions, as summarized in Table 1 , we obtained over 4\% PCE and $6.78 \%$ PCE after optimization with an additive $(1.3$ vol\% 1,8-octanedithiol). The improved PCE can be attributed to an increased molecular weight $\left(\mathrm{M}_{\mathrm{n}}=23000 \mathrm{~g} / \mathrm{mol}\right)$ and enhanced solubility. For example, dissolving PBT8PT $(10 \mathrm{mg})$ in $o$-dichlorobenzene $(1.5 \mathrm{~mL})$ yields the best performance with a 120-nm-thick active layer which is $40 \mathrm{~nm}$ thicker than the devices fabricated from PBTPDO.

In order to verify how the OPV performance depends on the fabrication process, the obtained active layers were characterized with energy filtered transmission electron microscopy (EF-TEM). As shown in Figure 5, both zero-loss and energy-filtered $(27 \pm 3 \mathrm{eV})$ conditions were conducted to interpret the film morphology of PBT8PT/PCBM[70], because less conducting PBT8PT is bright under the zero-loss state and becomes dark under the energy-filtered state. Based on the diffraction patterns and the obtained EF-TEM images, the thermally annealed PBT8PT/ PCBM[70] device exhibits better crystallinity than the nonannealed device, which facilitates carrier transport. However, the thermally annealed device exhibits poor performance as $V_{o c}$ and $J_{\text {sc }}$ are reduced. As described in our previous work, a fast drying condition does not allow enough time for the phase separation between PBT8PT and PCBM[70], resulting in an island or a culde-sac domain including a narrow intermolecular distance. This causes a strong recombination, and ultimately decreases $V_{o c}$ and impairs the charge collection efficiency. ${ }^{[31]}$ In case of the additive assisted annealed device, it exhibits a much stronger crystalline phase of PBT8PT, compared to the nonannealed condition, though both samples were evenly treated except for the additive. We believe that the additive removes PCBM[70] from polymerrich domains through its orthogonal solubility, which facilitates PBT8PT aggregation because small size PCBM[70] acts as a plasticizer in the polymer matrix. Thus, the additive assisted annealing method is useful to produce a percolation pathway including a highly crystalline polymer domain. ${ }^{[13]}$ As shown in Figure 4c, absorption spectra of the fabricated OPV devices under different process conditions are similar, but the additive assisted annealed device exhibited higher external quantum efficiency (EQE) through overall wavelength and an enhanced fill factor (FF) (Table 1 and Figure 4d). This implies that the additive assisted annealed device has a superior charge dissociation and carrier collection pathway, because the absorption and the dissociation energy offset are likely similar, regardless of the device fabrication process for the same material composition. Interestingly, when the additive assisted annealed device was thermally treated further it did not exhibit photovoltaic behavior. As shown in Figure $5 \mathrm{~g}$, the pristine crystalline phase generated by the additive assisted annealing manifested in Figure 5e was deteriorated 


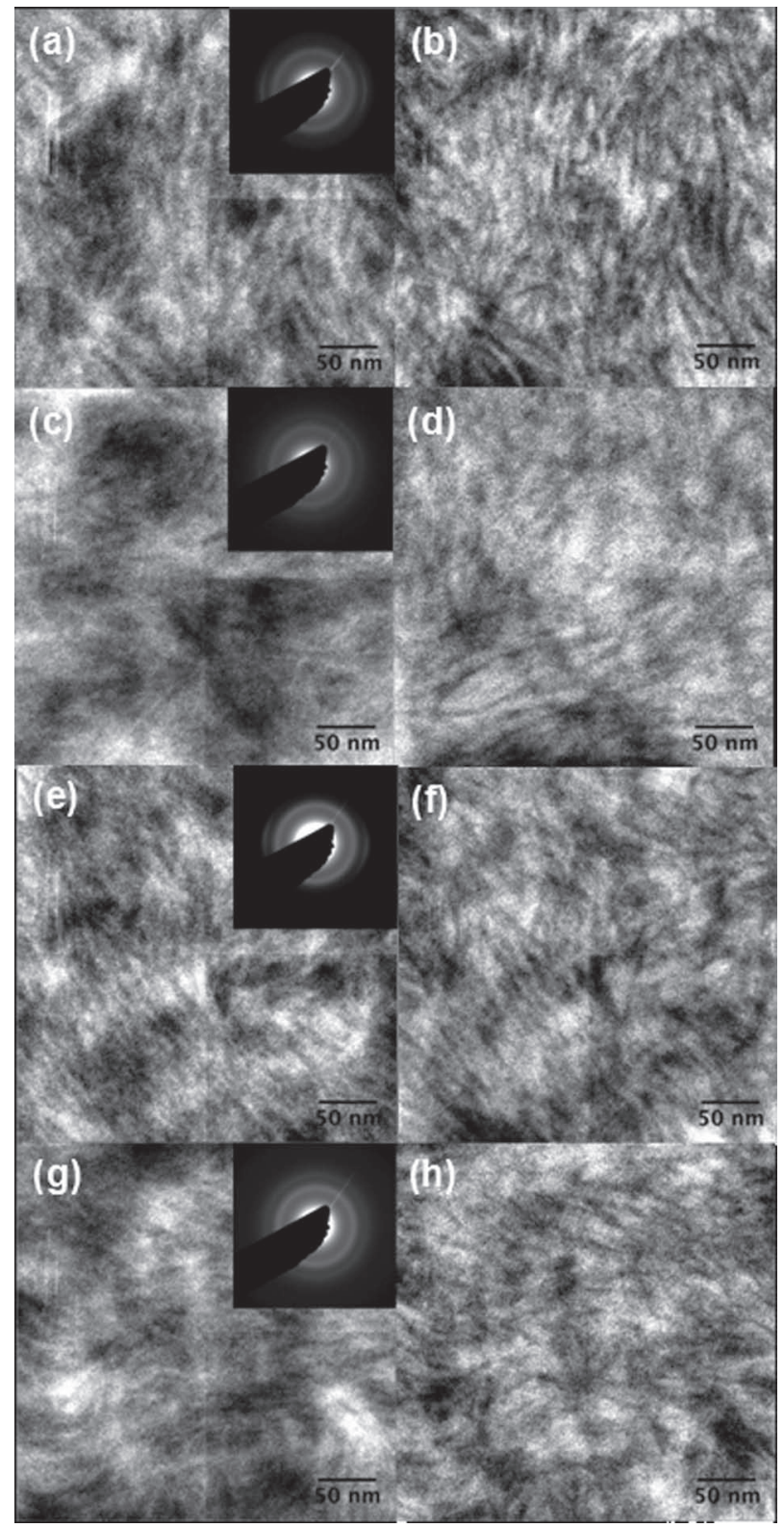

Figure 5. EF-TEM images of active layers of the PBT8PT/PCBM[70] film formed under different fabrication conditions. a,b) thermally annealed; c,d) non-annealed; e,f) additive assisted annealed; and g,h) thermally annealed after additive assisted annealing. Insets indicate diffraction patterns.

by an additional thermal treatment. It is probable that the remnant additive causes PCBM[70] to rediffuse into PBT8PT domains during the additional thermal annealing, because the additive has a decent solubility towards PBT8PT at the applied thermal annealing condition $\left(130^{\circ} \mathrm{C}\right.$ for $\left.15 \mathrm{~min}\right)$.

\section{Conclusions}

A series of CPs with alternating D-A monomer units to predictably modulate the HOMO and the LUMO of CPs were successfully synthesized. The CPs' energy levels were modified by changing the electron withdrawing power of the acceptor monomers, while using the same donor monomer. Stronger electron withdrawing groups in the acceptor monomer result in lower HOMO and LUMO energy levels in the resulting CP. The LUMO level drops more rapidly than the HOMO level, which ultimately produces a narrowed band-gap. Furthermore, the shift in the HOMO level of the CP compared to the midpoint value between the HOMO levels of the constituent monomers, $\Delta E_{\mathrm{H}}$, converges towards a constant value regardless of the difference in the monomers' HOMO values. The same holds true for the LUMO as well. This trend is universally applicable to similarly structured CPs, with different constant values for different CP conformations. Conversely, this method may be used to predict CP energy levels, given a set of monomers' energy levels. We demonstrate these predictive capabilities using the example of PBT8PT. The energy levels of the newly obtained PBT8PT show good agreement with the anticipated energy levels from the suggested energy level correlation between CP and its own monomers. Using OPV devices fabricated with the obtained CPs, we verify that $V_{\text {oc }}$ increases as CP's HOMO level decreases and $J_{\text {sc }}$ increases as CP's absorption approaches a high photon flux region. After OPV device optimization with the additive, the PBT8PT/PCBM[70] blend achieved 6.78\% PCE mostly due to the combined effects including enlarged molecular weight, enhanced solubility and an improved charge collection pathway. Finally, the morphology of the active layers generated by different fabrication conditions was characterized with EF-TEM. Both the thermal and additive treatments are useful to enhance the crystallinity of PBT8PT, but the thermally annealed device cannot utilize the advantage of the enhanced crystallinity because of the accompanied strong recombination. The additive assisted annealed device exhibits highly crystalline PBT8PT, which results in best OPV performance among the applied device fabrication conditions.

\section{Experimental Section}

Synthesis: All starting materials were purchased from commercial suppliers (Aldrich and Fisher Sci.). Detailed synthetic procedures are summarized in the Supporting Information and synthesized compounds were fully characterized with ${ }^{1} \mathrm{H}-\mathrm{NMR}$ and GC-mass spectroscopy. Molecular weights of the final polymers were measured with gel permeation chromatography (GPC) using polystyrene calibration standards.

Molecular Geometry Calculations: Electronic structure calculations and spatial relaxation of the molecules were performed using Gaussian03. ${ }^{[32]}$ Pre-optimizations of the molecules were carried out using AM1 semiempirical quantum chemistry model. ${ }^{[33]}$ The resulting molecular configurations were then further optimized in the density functional theory (DFT) framework. We used B3LYP as the exchange-correlation functional, that is, a Hartree-Fock-DFT hybrid where the exchange energy is explicitly calculated using the Hartree-Fock approach. ${ }^{\left[{ }^{[3]}\right.}$ The molecular geometries were optimized in the Cartesian coordinate system without any symmetry (maximum degrees of freedom) using 6-31C* contracted Gaussian basis set with polarization functions. ${ }^{[35,36]}$

Cyclic Voltammetry Measurements: $\mathrm{Ag} / \mathrm{AgNO} 3$ served as the pseudo reference electrode, ferrocene was used for calibration, and the supporting electrolyte was tetrabutylammonium hexafluorophosphate in acetonitrile. Oxidation and reduction potentials of each CP were directly measured after casting them on top of ITO coated glass.

OPV Device Fabrication: PCBM[70] was purchased from SES research and used without further purification. After dissolving obtained CPs $(10 \mathrm{mg})$ and PCBM[70] $(10 \mathrm{mg})$ into o-dichlorobenzene $(2.0 \mathrm{~mL})$ and 
1,8-octanedithiol $(26 \mu \mathrm{L})$, the mixture was filtered with a syringe filter ( $0.45 \mu \mathrm{m}$ pore diameter) and used to form the active layer by means of spin casting. In case of PBT8PT, $1.5 \mathrm{~mL}$ of $o$-dichlorobenzene and $20 \mu \mathrm{L}$ of 1,8-octanedithiol were used for the blend solution. ITO-coated glass was cleaned with acetone and IPA followed by UV ozone treatment for 10 min. PEDOT:PSS (Baytron PH 500) was spin cast on the substrate and baked at $140{ }^{\circ} \mathrm{C}$ for $15 \mathrm{~min}$. Different polymer/PCBM[70] blend solutions were spin cast at $700 \mathrm{rpm}$ for $30 \mathrm{~s}$ and used for metal electrode evaporation without further treatment. Final devices were fabricated by depositing a 1-nm-thick LiF and a $70 \mathrm{~nm}$ Al layer $\left(9.6 \mathrm{~mm}^{2}\right)$ sequentially under $5 \times 10^{-7}$ Torr $(1$ Torr $=133.32 \mathrm{~Pa})$. All devices were characterized under nitrogen and the typical illumination intensity was $100 \mathrm{~mW} / \mathrm{cm}^{2}$ (AM 1.5G Oriel solar simulator).

EFTEM Measurements: The morphology of blends was examined using energy-filtered transmission electron microscopy (EFTEM) in a JEOL 2100F TEM, equipped with a Gatan \#863 Tridiem imaging filter (GIF), operated at an acceleration voltage of $200 \mathrm{kV}$. The films were floated from deionized water and picked up onto copper grids (Ted Pella, Inc.) prior to TEM analysis. EFTEM spectral images were collected using an energy slit width of $8 \mathrm{eV}$ and a step width of $0.8 \mathrm{eV}$, from -5 to $40 \mathrm{eV}$, covering the zero-loss regime and plasmon-loss regime on the energy loss spectra.

\section{Supporting Information}

Supporting Information is available from the Wiley Online Library or from the author.

\section{Acknowledgements}

We thank Dr. Chang-Gua Zhen for the energy level calculation of applied monomers. This research was supported as part of the Center for Solar and Thermal Energy Conversion, an Energy Frontier Research Center funded by the U.S. Department of Energy, Office of Science, Office of Basic Energy Sciences under Award No. DE-SC0000957.

Received: May 22, 2012 Published online: September 14, 2012

[1] H.-Y. Chen, J. Hou, S. Zhang, Y. Liang, G. Yang, Y. Yang, L. Yu, Y. Wu, G. Li, Nat. Photonics 2009, 3, 649

[2] H. Zhou, L. Yang, A. C. Stuart, S. C. Price, S. Liu, W. You, Angew. Chem. Int. Ed. 2011, 50, 2995.

[3] Y. Zhang, J. Zou, H.-L. Yip, K.-S. Chen, D. F. Zeigler, Y. Sun, A. K.-Y. Jen, Chem. Mater. 2011, 23, 2289.

[4] C. Piliego, T. W. Holcombe, J. D. Douglas, C. H. Woo, P. M. Beaujuge, J. M. J. Fréchet, J. Am. Chem. Soc. 2010, 132, 7595.

[5] S. H. Park, A. Roy, S. Beaupré, S. Cho, N. Coates, J. S. Moon, D. Moses, M. Leclerc, K. Lee, A. J. Heeger, Nat. Photonics 2009, 3, 297

[6] Y. Liang, Z. Xu, J. Xia, S.-T. Tsai, Y. Wu, G. Li, C. Ray, L. Yu, Adv. Mater. 2010, 22, E135.

[7] A. Tada, Y. Geng, Q. Wei, K. Hashimoto, K. Tajima, Nat. Mater. 2011, 10,450.

[8] S. K. Pal, T. Kesti, M. Maiti, F. Zhang, O. Inganäs, S. Hellström, M. R. Andersson, F. Oswald, F. Langa, T. Österman, T. Pascher, A. Yartsev, V. Sundström, J. Am. Chem. Soc. 2010, 132, 12440.

[9] J.-L. Brédas, J. E. Norton, J. Cornil, V. Coropceanu, Acc. Chem. Res. 2009, 11, 1691

[10] D. H. Wang, D. Y. Kim, K. W. Choi, J. H. Seo, S. H. Im, J. H. Park, O. O. Park, A. J. Heeger, Angew. Chem. Int. Ed. 2011, 50, 5519.
[11] J. K. Lee, W. L. Ma, C. J. Brabec, J. Yuen, J. S. Moon, J. Y. Kim, K. Lee, G. C. Bazan, A. J. Heeger, J. Am. Chem. Soc. 2008, 1303619.

[12] M. D. Perez, C. Borek, S. R. Forrest, M. E. Thompson, J. Am. Chem Soc. 2009, 131, 9281

[13] J. Peet, J. Y. Kim, N. E. Coates, W. L. Ma, D. Moses, A. J. Heeger, G. C. Bazan, Nat. Mater. 2007, 6, 497.

[14] N. Blouin, A. Michaud, M. Leclerc, Adv. Mater. 2007, 19, 2295.

[15] H. A. M. van Mullekom, J. A. J. M. Vekemans, E. E. Havinga, E. W. Meijer, Mater. Sci. Eng., A. 2001, 32, 1.

[16] L.-L. Chua, J. Zaumseil, J.-F. Chang, E. C.-W. Ou, P. K.-H. Ho, H. Sirringhaus, R. H. Friend, Nature 2005, 434, 194.

[17] C.-H. Chen, C.-H. Hsieh, M. Dubosc, Y.-J. Cheng, C.-S. Hsu, Macromolecules 2010, 43, 697.

[18] K. C. Park, L. R. Dodd, K. Levon, T. K. Kwei, Macromolecules 1996 29, 7149.

[19] M. Leclerc, Adv. Mater. 1999, 11, 1941.

[20] T. Huser, M. Yan, L. J. Rothberg, Proc. Natl. Acad. Sci. USA 2000, 97, 11187.

[21] Y. Liang, Y. Wu, D. Feng, S.-T. Tsai, H.-J. Son, G. Li, L. Yu, J. Am. Chem. Soc. 2009, 131, 56.

[22] H. J. Son, F. He, B. Carsten, L. Yu, J. Mater. Chem. 2011, 21, 18934

[23] C. Risko, M. D. McGehee, J.-L. Brédas, Chem. Sci. 2011, 2, 1200.

[24] A. Ajayaghosh Chem. Soc. Rev. 2003, 32, 181.

[25] M. C. Scharber, M. Koppe, J. Gao, F. Cordella, M. A. Loi, P. Denk, M. Morana, H.-J. Egelhaaf, K. Forberich, G. Dennler, R. Gaudiana, D. Waller, Z. Zhu, X. Shi, C. J. Brabec, Adv. Mater. 2010, 22, 367.

[26] J. C. Bijleveld, M. Shahid, J. Gilot, M. M. Wienk, R. A. J. Janssen, Adv. Funct. Mater. 2009, 19, 3262.

[27] W. Yue, Y. Zhao, S. Shao, H. Tian, Z. Xie, Y. Geng, F. Wang, J. Mater. Chem. 2009, 19, 2199.

[28] K. M. Noone, E. Strein, N. C. Anderson, P.-T. Wu, S. A. Jenekhe, D. S. Ginger, Nano Lett. 2010, 10, 2635.

[29] W. Ma, J. Y. Kim, K. Lee, A. J. Heeger, Macromol. Rapid Commun. 2007, 28, 1776.

[30] H. Zhou, L. Yang, S. Xiao, S. Liu, W. You, Macromolecules 2010, 43, 811.

[31] B.-G. Kim, E.J. Jeong, H. J. Park, D. Bilby, L. J. Guo, J. Kim, ACS Appl. Mater. Interfaces 2011, 3, 674.

[32] Gaussian 03, Revision C.02, M. J. Frisch, G. W. Trucks, H. B. Schlegel, G. E. Scuseria, M. A. Robb, J. R. Cheeseman Jr., J. A. Montgomery, T. Vreven, K. N. Kudin, J. C. Burant, J. M. Millam, S. S. lyengar, J. Tomasi, V. Barone, B. Mennucci, M. Cossi, G. Scalmani, N. Rega, G. A. Petersson, h. Nakatsuji, M. Hada, M. Ehara, K. Toyota, R. Fukuda, J. Hasegawa, M. Ishida, T. Nakajima, Y. Honda, O. Kitao, H. Nakai, M. Klene, X. Li, J. E. Knox, H. P. Hratchian, J. B. Cross, V. Bakken, C. Adamo, J. Jaramillo, R. Gomperts, R. E. Stratmann, O. Yazyev, A. J. Austin, R. Cammi, C. Pomelli, J. W. Ochterski, P. Y. Ayala, K. Morokuma, G. A. Voth, P. Salvador, J. J. Dannenberg, V. G. Zakrzewski, S. Dapprich, A. D. Daniels, M. C. Strain, O. Farkas, D. K. Malick, A. D. Rabuck, K. Raghavachari, J. B. Foresman, J. V. Ortiz, Q. Cui, A. G. Baboul, S. Clifford, J. Cioslowski, B. B. Stefanov, G. Liu, A. Liashenko, P. Piskorz, I. Komaromi, R. L. Martin, D. J. Fox, T. Keith, M. A. Al-Laham, C. Y. Peng, A. Nanayakkara, M. Challacombe, P. M. W. Gill, B. Johnson, W. Chen, M. W. Wong, C. Gonzalez, J. A. Pople, Gaussian, Inc., Wallingford CT, USA 2004

[33] J. J. P. Stewart, J. Comput. Chem. 1989, 10, 209.

[34] A. D. Becke, J. Chem. Phys. 1993, 98, 5648.

[35] M. M. Francl, W. J. Pietro, W. J. Hehre, J. S. Binkley, M. S. Gordon, D. J. Defrees, J. A. Pople, J. Chem. Phys. 1982, 77, 3654.

[36] W. J. Hehre, R. Ditchfie, J. A. Pople, J. Chem. Phys. 1972, 56, 2257. 